\title{
Young and Early Career Investigators: Report from a Global HIV Vaccine Enterprise Working Group
}

\author{
Dan H Barouch ${ }^{1}$, 13, Thumbi Ndung' $^{2}{ }^{2,13}$, Galit Alter ${ }^{3}$, Timothy Cardozo ${ }^{4}$, Bhavna Chohan ${ }^{5}$, Jacques Fellay ${ }^{6}$, \\ Nicole Frahm ${ }^{7}$, Jonathan Fuchs ${ }^{8}$, Al Leslie ${ }^{9}$, Yong Liu ${ }^{10}$, Penny Moore ${ }^{11}$ \& Raul Rabadan ${ }^{12}$
}

\section{A. SETTING THE STAGE}

The scientific challenges facing HIV-1 vaccine development are unprecedented in the history of vaccinology. As a result, investigators, funders, and other stakeholders generally agree that "game-changing" ideas are required. While innovation can certainly arise from investigators at all career stages, young and early-career investigators, defined as those under 40 years of age or within 10 years of their final degree or clinical training, are especially key contributors of novel and transformative ideas. Young and early-career investigators bring energy, enthusiasm, and fresh perspectives that are unbiased by prevailing dogma and that are essential to scientific progress.

Demographic analysis supports the premise that transformative new ideas often originate with young and early-career investigators ${ }^{1}$. Nobel laureates Harold Varmus, David Baltimore, James Watson and Dorothy Hodgkin all made their groundbreaking discoveries before the age of 30. Charles Darwin conceived the theory of natural selection when he was 29. Albert Einstein published his papers about special relativity and quantum mechanics when he was 26 . Isaac Newton began developing calculus and the law of gravitation at 25 . This anecdotal evidence, as well as demographic analysis of Nobel laureates ${ }^{1}$, suggests that young and early-career investigators are a major source of innovative ideas that often drive key paradigm shifts in all areas of science.

\section{B. CHALLENGES AND OPPORTUNITIES}

The Global HIV Vaccine Enterprise established a committee of Young and Early Career Investigators (YECIs) in 2008 to define key bottlenecks currently faced by YECIs in the HIV-1 vaccine field and to suggest potential solutions. Members of this committee were recruited from both the developed and the developing world and represent a wide diversity of backgrounds and scientific interests.

The YECI committee (http://www.vaccineenterprise.org/content/ young-and-early-career-investigators-yeci), organized a series of meetings and consultations in 2008 and 2009 to solicit broad perspectives from young investigators, senior investigators, funders, and other stakeholders to inform the development of this report. Open sessions were held at the 2008 International AIDS conference in Mexico City, the AIDS Vaccine 2008 conference in Cape Town, the 2009 Keystone Symposium on HIV Vaccines, and the AIDS Vaccine 2009 conference in Paris. Additional information was obtained through surveys, open discussions, small group meetings, and online communications.

The major obstacles facing YECIs in the HIV-1 vaccine field that were identified can be summarized as follows:

(i) The perception of HIV-1 vaccine research as saturated, competitive, empiric, and unproductive may inhibit the recruitment of the best and the brightest YECIs to the field.

(ii) The increasingly large-scale, complex, and interdisciplinary nature of HIV-1 vaccine research requires multidisciplinary training with broad knowledge in multiple areas. This will require redefining traditional mentorship, training, and career development pathways.

(iii) The lack of access to key datasets and resources may impede YECI research and creativity.

(iv) YECIs currently face unprecedented challenges in securing initial funding to launch what should be one of the most creative phases of their careers. These funding challenges are further exacerbated by the lack of sufficient numbers of faculty positions and protected career development pathways at academic institutions ${ }^{2}$. From 1980 to 2007, the average age of principal investigators funded by the US National Institutes of Health (NIH) has increased markedly (Fig. 1a), and the age of first-time NIH grant recipients has risen to an all-time high of 41 years of age (Fig. 1b). These data suggest that the turnover of resources, laboratory space, and academic positions to young investigators is inadequate.

(v) Moreover, visibility and leadership representation of YECIs in the HIV-1 vaccine field represent further challenges for their development as independent investigators.

(vi) Finally, YECIs who live and work in the developing world, where most of the new HIV-1 infections occur, represent a critical and underutilized reservoir of skill, innovation, and determination. YECIs in the developing world face profound logistic, funding, mentorship, institutional, and career development challenges that require particular attention.

In this report, we offer potential solutions to these problems. Our primary goal is not to advocate for increased support for YECIs per $s e$, but rather to ensure that YECIs are able to contribute fully to the development of an HIV-1 vaccine. The success of the HIV vaccine effort will undoubtedly depend on the innovation, enthusiasm, and engagement of the next generation of investigators.

\footnotetext{
${ }^{1}$ Beth Israel Deaconess Medical Center, USA. ${ }^{2}$ University of KwaZulu-Natal, South Africa. ${ }^{3}$ Ragon Institute, USA. ${ }^{4}$ New York University School of Medicine, USA ${ }^{5}$ University of Nairobi, Kenya. ${ }^{6}$ Duke University, USA. ${ }^{7}$ Fred Hutchinson Cancer Research Center, USA. ${ }^{8}$ University of California San Francisco, USA. ${ }^{9}$ University of Oxford, UK. ${ }^{10}$ Center for Disease Control and Prevention, China. ${ }^{11}$ National Institute for Communicable Diseases, South Africa. ${ }^{12}$ Columbia University, USA.- $\bar{\lambda}$ 13 Working group chairs.
} 


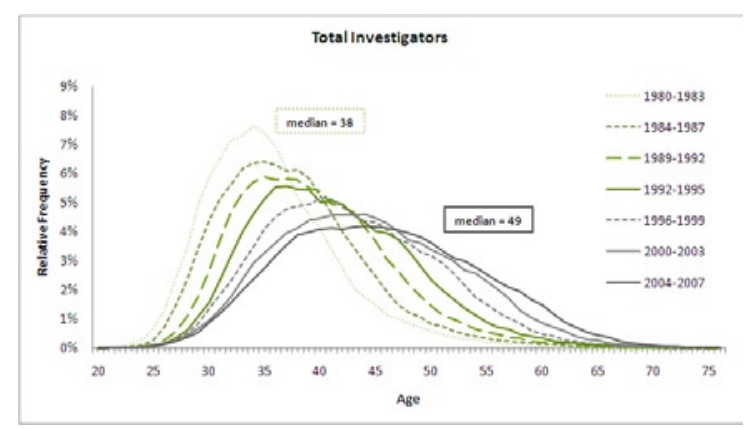

b

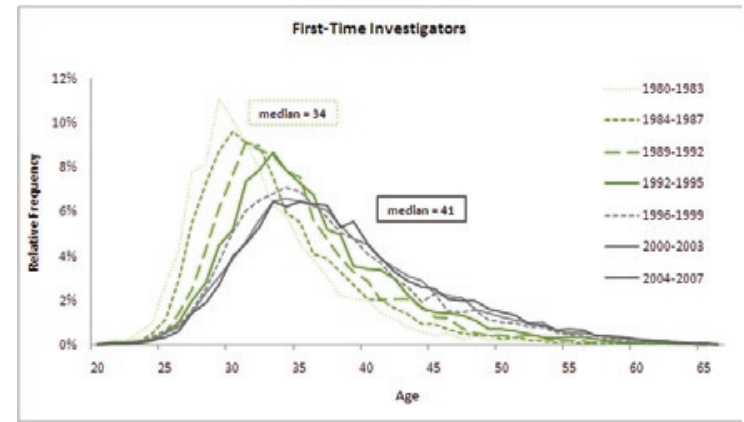

\section{RECOMMENDATIONS}

To address these challenges, we recommend the development of a multi-component YECI Initiative as part of the 2010 Scientific Strategic Plan of the Global HIV Vaccine Enterprise. This YECI Initiative involves a coordinated strategy (Table 1) to establish a new infrastructure and culture that will best enable YECIs to develop and to explore new ideas in the HIV-1 vaccine field. The current paucity of independently funded YECIs may represent one of the most critical impediments to innovation in the HIV-1 vaccine field.

\section{Mentorship}

We first suggest that outstanding mentorship in the HIV-1 vaccine field should be recognized and encouraged by establishing Excellence in Mentoring Awards. These awards would be chosen by YECIs and would include mentors from both the developed and developing worlds. Thes could be presented during the plenary sessions of the annual AIDS Vaccine Conferences. Academic institutions should also be encouraged to increase their emphasis on mentorship as a criterion for promotion. Our goal is to ensure that these Mentorship Awards are highly prestigious awards that recognize the full responsibility of senior colleagues not only to conduct their own research but also to ensure the success of the next generation of investigators.

\section{Career Development}

Training and career development pathways urgently need to be reexamined in the face of the profoundly changing way in which biomedical research is conducted today. To that end, we recommend a number of discrete action items: First, increased numbers of transportable postdoctoral salary support fellowships would allow trainees the flexibility to define and to explore their own ideas. Second, more comprehensive multidisciplinary training opportunities could be provided, such as expanding the popular satellite workshops that are focused on a variety of scientific and career development topics and that are held in conjunction with major scientific conferences. The goal of these training sessions would be to provide more comprehensive educational experiences without increasing the overall length of postdoctoral training. Third,
Figure 1 Distribution of NIH research project grants by age between 1980 and 2007 (http://grants.nih.gov). (a) Aging population of all investigators receiving $\mathrm{NIH}$ research project grants resulting in the median age increasing from 38 years in 1980 to 49 years in 2007. (b) Increasing age of first-time recipients of $\mathrm{NIH}$ research project grants. The median age of a first-time investigator has increased from 34 years in 1980 to 41 years in 2007. (First-time investigators are defined as principal investigators who have not previously competed successfully for a major $\mathrm{NIH}$ research grant award.)

increasing the number of start-up funds and faculty positions for YECIs would greatly facilitate the difficult transition from postdoctoral fellow to independent investigator. Fourth, a centralized, online resource could be created and hosted by the Enterprise to support a global community of YECIs by providing up-to-date information about funding and employment opportunities, mentors, training courses, workshops, and other activities. This resource could also serve as a forum for discussion and a virtual meeting place for YECIs.

To address the particular needs of YECIs in the developing world, we recommend the creation of additional regional Centers of Excellence at institutions that have the necessary infrastructure and critical mass. Existing Centers of Excellence in developing countries should also be strengthened. Long-term funding to such Centers could be linked to ongoing recruitment and development of YECIs. These Centers would be expected to provide mentorship, training, research and networking infrastructure, exposure, and solid career development paths and would also include faculty partnerships and YECI exchange programs with research institutions in the developed world. Coordinated establishment, funding, and local ownership and leadership of these Centers would be crucial to ensure sustainability.

\section{Data Sharing and Open Access}

We also recommend increased open access to data and reagents in the HIV-1 vaccine field to ensure that YECIs (as well as others) can contribute

Table $1_{\Lambda}$ Summary of priorities and recommendations of the YECI Initiative.

Mentorship, Career Development, and Open Access

1. Establish Excellence in Mentoring Awards

2. Strengthen training and career development pathways

a. Increase numbers of transportable postdoctoral salary support fellowships

b. Offer more comprehensive multidisciplinary training opportunities

c. Increase availability of start-up funds and faculty positions

d. Create a centralized global electronic YECl community

3. Create and enhance regional Centers of Excellence in the developing world 4. Increase open access to data and resources

Novel Funding Approaches

1. Establish funds to recruit YECls from traditionally non-HIV-1 fields

2. Develop targeted initial independent grant programs for $\mathrm{YECls}$ both in the developed and in the developing world

3. Award research grants from YECls and established investigators at similar success rates

4. Set quantitative targets for funding of $\mathrm{YECI} \mathrm{PIs}$ in the context of large consortia

\section{Visibility and Leadership Representation}

1. Encourage a cultural shift in which YECls would have increased visibility and greater leadership representation

2. Set quantitative targets for conference organizers to include YECIs as invited speakers at international conferences

3. Include YECIs on peer review panels for grant proposals, editorial boards for journals, and scientific leadership groups of major consortia and other decision-making bodies 
fully to the collaborative HIV vaccine efforts. Arguably data obtained in whole or in part with public or philanthropic funds should be made openly accessible as soon as practically possible. To facilitate this goal, a centralized HIV-1 database resource could be created to collate and to provide open access to datasets and to platforms that facilitate rapid sharing of methodologies and reagents. In addition, we endorse open access publication and the creation of a pre-print server of peer-reviewed HIV-1 vaccine research.

\section{Novel Funding Approaches}

We next suggest a series of approaches to facilitate improved independent funding opportunities for YECIs. First, a fund could be established to provide research support to YECIs from traditionally non-HIV fields who are interested in becoming engaged in HIV-1 vaccine research. The goal would be to recruit and to retain the best young minds and to increase the breadth of expertise in the HIV-1 vaccine field. Second, targeted initial independent grant programs could be developed for YECIs both in the developed and in the developing world to facilitate transitions from postdoctoral to independent investigator. Third, funders should strive to award research grants to YECIs and established investigators at similar success rates to compensate for potential biases in the peer review process. Fourth, quantitative targets should be set for funding of YECI PIs in the context of programs and large consortia. We would suggest, for example, that at least $20 \%$ of research funds in large consortia should be targeted to YECI PIs if proposed concepts are scientifically meritorious. This strategy would increase funding opportunities for YECIs as well as help solidify their leadership roles in the context of consortia.

\section{Visibility and Leadership Representation}

Finally, we advocate for a cultural shift in which YECIs would have increased visibility and enhanced leadership role in the HIV-1 vaccine field. For example, we encourage quantitative targets for conference organizers to include YECIs as invited speakers. We recommend that at least $25 \%$ of plenary lectures and $50 \%$ of all oral presentations at international conferences be given by YECIs. We also suggest that peer review panels for grant proposals, editorial boards for journals, and scientific leadership groups of major consortia and other decision-making bodies should include YECIs. Particular emphasis should be placed on including YECIs from the developing world.

\section{CONCLUSIONS}

The multifaceted YECI Initiative proposed in this report is aligned with the overall goals of the 2010 Scientific Strategic Plan of the
Global HIV Vaccine Enterprise and in particular the strategic need to attract and to develop YECIs to harness the ongoing revolution in the biomedical sciences. In particular, YECIs represent a critically important source of innovation for the field ${ }^{3,4}$. The present report provides a framework of the key challenges faced by YECIs in the HIV-1 vaccine field and offers potential approaches to solving these problems.

A number of meaningful steps have already been taken to address these problems since the Enterprise first championed the strategic importance of YECIs for the HIV-1 vaccine field. For example, by increasing the research project grant payline for new investigators, the National Institute of Allergy and Infectious Diseases (NIAID) achieved comparable levels of funding for new and established investigators in 2009. The Wellcome Trust has recently articulated a new policy that focuses on people rather than projects with a particular emphasis on young investigators. The Center for HIV-AIDS Vaccine Immunology (CHAVI) and the Collaboration for AIDS Vaccine Discovery (CAVD) have established programs that regularly recognize young investigators, and NIAID has committed new funding that has been awarded by ${ }_{\Sigma}$ IV Vaccine Trials Network (HVTN), and CHAVI to early-stage investigators with projects that aim to bridge the gaps between nonhuman primate and clinical research. In addition, conference organizers appear to be placing a higher priority on inviting YECI speakers at regional and international conferences. These and other programs suggest the beginning of an important cultural shift aimed at encouraging YECIs and ensuring that their creativity and innovation will help to drive the HIV-1 vaccine field forward. We strongly applaud this trend, although current efforts are still inadequate, particularly in the developing world. We believe that the recommendations proposed in this report will address many of the challenges currently faced by YECIs and by so doing will accelerate the development of an HIV-1 vaccine.

\section{ACKNOWLEDGMENTS}

We acknowledge generous advice and assistance from Alan Bernstein, Amapola Manrique, Yegor Voronin, and the Global HIV Vaccine Enterprise.

1. Dietrich, A. \& Srinivasan, N. The optimal age to start a revolution. J. Creat. Behav. 41, 54-74 (2007).

2. Kaiser, J. Biomedical research: the graying of nih research. Science 322, 848-849 (2008).

3. American Academy of Arts and Sciences. ARISE - Advancing Research In Science and Engineering: Investing in Early-Career Scientists and High-risk, High Reward Research. <http://www.amacad.org/arisefolder/default.aspx> (Cambridge, Massachussetts, 2008).

4. Kaiser, J. Zerhouni's parting message: make room for young scientists. Science $\mathbf{3 2 2}$ 834-835 (2008). 